\title{
Attenuation of disturbances introduced by dynamic links in precision motion systems using model-based observers
}

\author{
M. Hoogerkamp ${ }^{\mathrm{a}, \mathrm{b}, 1}$, R.R. Waiboer ${ }^{\mathrm{a}, *}$, J. van Dijk ${ }^{\mathrm{b}}$, R.G.K.M. Aarts ${ }^{\mathrm{b}}$ \\ ${ }^{a}$ Mechatronic Systems Development, ASML Netherlands B.V., P.O. Box 324, 5500 AH Veldhoven, The Netherlands \\ ${ }^{\mathrm{b}}$ Department of Engineering, University of Twente, P.O. Box 217, 7500 AE Enschede, The Netherlands
}

\section{A R T I C L E I N F O}

\section{Article history:}

Received 30 August 2013

Revised 11 April 2014

Accepted 29 April 2014

Available online 16 May 2014

\section{Keywords:}

Disturbance observer

Kalman filter

Internal model control

Wafer stage

Cable schlepp

Experimental validation

\begin{abstract}
A B S T R A C T
This paper presents an extension to the Unknown Input Disturbance Observer (UIDO) and the Disturbance Estimation Filter (DEF). This extension enables the inclusion of the mechanics of dynamic links to the observer model, in order to attenuate the specific disturbances introduced by those dynamic links. A design method of the state space feedback gain based on the dynamics, and an observer gain based on basic Kalman filter theory is given. It is shown how the observer is designed for a practical example; the cable schlepp within the wafer stage of a lithography machine. Using a simple model of the cable schlepp the disturbance observer design has been validated with an experiment on an actual machine.
\end{abstract}

(c) 2014 Elsevier Ltd. All rights reserved.

\section{Introduction}

In high-speed nano-scale positioning systems, such as the stages used in the wafer scanning industry shown in Fig. 1, highspeed motion is combined with nano-scale tracking precision. In terms of achieving servo performance, the combination of both speed and accuracy puts heavy demands on the control systems and design. The amount of disturbance rejection of the control system is limited due to the fact that the servo bandwidth is restricted by the elastic modes of the wafer or reticle positioning system [1]. A main source of disturbance forces are the so-called dynamic links [2]. These are for instance:

- hoses for transportation of coolant and gas, and

- wires and flexible printed circuit boards for electrical power and sensor signals.

With the cross-links between stages, movement of one stage is linked to the other, and vibrations of the dynamic links itself introduce disturbances to the stages. One example of a dynamic link is the cable schlepp attached to the long stroke of a wafer stage, as

\footnotetext{
* Corresponding author. Tel.: +31 402682137.

E-mail addresses: meinko.hoogerkamp-mhqy@asml.com (M. Hoogerkamp), rob. waiboer@asml.com (R.R. Waiboer), j.vandijk@utwente.nl (J. van Dijk), r.g.k.m. aarts@utwente.nl (R.G.K.M. Aarts).

${ }^{1}$ Principal corresponding author.
}

shown in Fig. 2. Disturbances of the cable schlepp to the long stroke are a main cause of a long settling time of the long stoke, and improving this would allow for an increase of performance.

In order to achieve a reduction of the effect of disturbances on a controlled system, the Unknown Input (state space) Disturbance Observer (UIDO) was introduced [3]. Within this structure the plant model in the observer is augmented by an autonomous system that describes the disturbance acting on the plant. The observer is used to estimate the states of the plant and the disturbance force acting on it. The estimated disturbance force is then used as a feedback force signal so that the error introduced by the disturbance is attenuated. This observer does not control the full system, a feedback control loop, with for example a PID controller and a feed forward as shown in Fig. 3, is needed to achieve the desired performance for high performance stage control. The separation of tasks allows for the independent design of both the position controller and feed forward mechanism to achieve maximum performance, and the disturbance observer to enable maximum disturbance attenuation.

Model based observers allow for the use of prior knowledge of the system to be applied directly for disturbance attenuation, instead of modifying the response of controllers and input of feed forwards to achieve the same goal. The observer has the advantage over controller loop shaping or feed forward frequency input shaping as only a single design effort for both feedback and feed forward control is needed. 


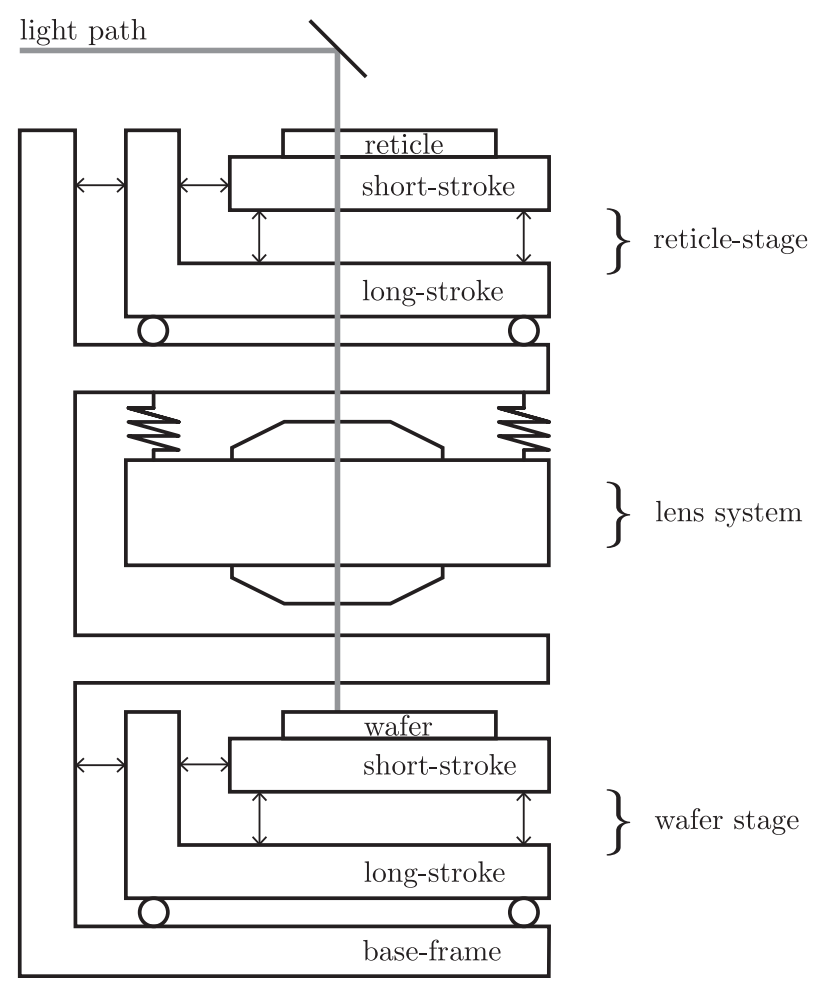

Fig. 1. Overview of the stages of a lithography machine.

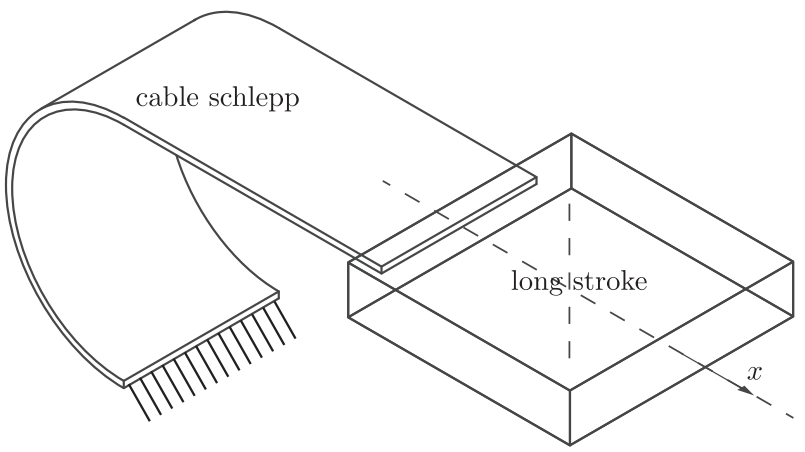

Fig. 2. Overview of a cable schlepp attached to the long stroke of the wafer stage. The length of the cable schlepp is in the order of a meter. For the purposes of this paper only moves in $x$-direction are considered.

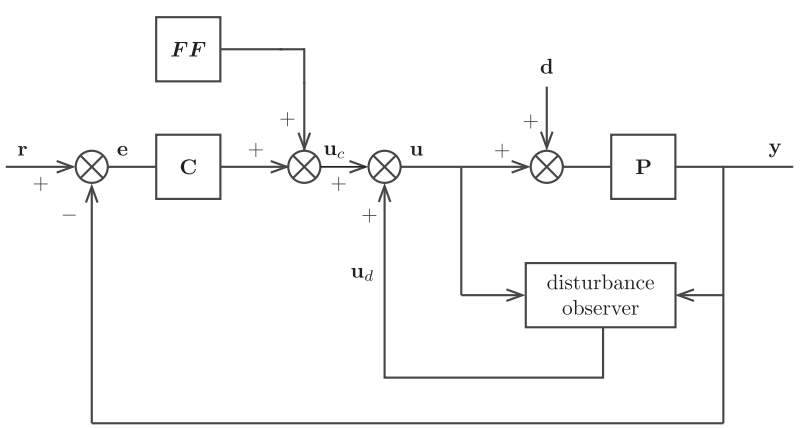

Fig. 3. Disturbance observer inside a control loop with the position controller $C$, feed forward $F F$ and the plant $P$.

It was shown that the UIDO is a specific implementation of the Disturbance Observer (DO) or Disturbance Estimation Filter (DEF)

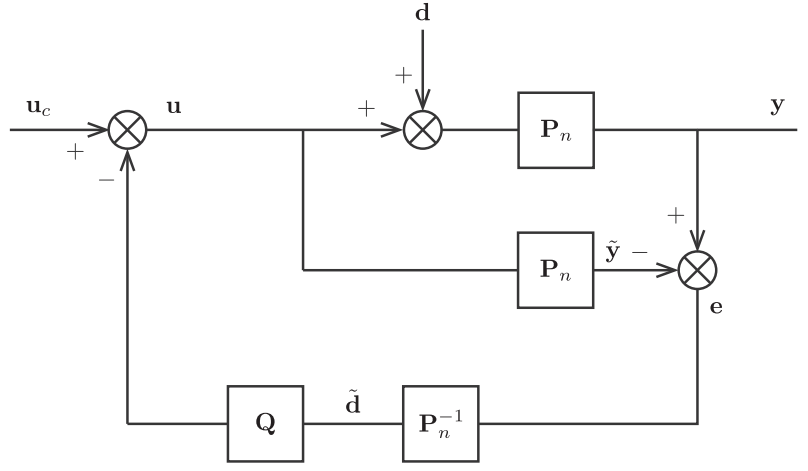

Fig. 4. General disturbance observer or disturbance estimation filter structure.

[4,5]. In Fig. 4 an implementation of the DEF structure is shown. By an inverse plant $\mathbf{P}_{n}^{-1}$ the disturbance force estimate $\tilde{\boldsymbol{d}}$ of $\mathbf{d}$ is reconstructed. With the filter $\mathbf{Q}$, the frequency content $\tilde{\mathbf{d}}$ is filtered such that stability of the observer is obtained. Fig. 4 emphasizes two general points of disturbance observers. The first is that disturbance estimation is effectively a plant inversion problem [6]. The second is that the source of the disturbance dynamics $\mathbf{d}$ is assumed not to be related to the input of the plant.

Both the UIDO and the more general DEF can be used to attenuate disturbances resulting from dynamic links. However, both do not use all information which can be gathered from the mechanics of the dynamic links. With application of analysis methods (e.g. FEM) for modeling dynamic links [7], more information about the nature of the disturbance is available which can be used to estimate and attenuate specific disturbances.

In this paper a method is presented which is an extension from both the DEF and UIDO, where the disturbance acting on a nominal plant is not assumed to be autonomous, but that the disturbance is an integrated part of the plant. The disturbance model is described with known parameters obtained from an analysis of the part of the system causing the disturbances on the nominal plant. Combined with an estimation of the states, this can be used to construct an estimation of the disturbance force on the nominal plant, creating an estimated input disturbance observer.

By considering this observer as an Internal Model Control (IMC) [8] problem, it is shown that the general disturbance observer problem is changed from dealing with the full input disturbance $d$ (Fig. 4) to creating robustness for a possible model mismatch. It is shown that integration of the disturbance dynamics in the observer allows attenuation of disturbances close to the bandwidth of the control system.

This paper is organized as follows. In Section 2, the properties of the disturbances are modeled. In Section 3 it is derived how this model can be used to estimate the disturbance forces. In Section 4 the disturbance attenuation problem is rewritten to an IMC problem. In Section 5 it is shown how robustness to modeling errors is obtained. An experimental setup is described in Section 6. The disturbance observer design is validated with experiments on a lithography machine in Section 7. Finally in Section 8 some conclusions are given.

\section{Modeling of the disturbance force}

A dynamic link disturbance can be modeled as a linearized system shown in Fig. 5 . The mass $m_{\text {stage }}$, for example representing a stage in a lithography machine, is position controlled in the degrees of freedom $x_{1}$ to $x_{n}$ using a feedback system which has a force input $F_{1}$ to $F_{n}$. Connected to the mass $m_{\text {stage }}$ with stiffness $k_{1}$ to $k_{n}$ and damping $c_{1}$ to $c_{n}$ are (smaller) masses $m_{d, 1}$ to $m_{d, n}$ with 


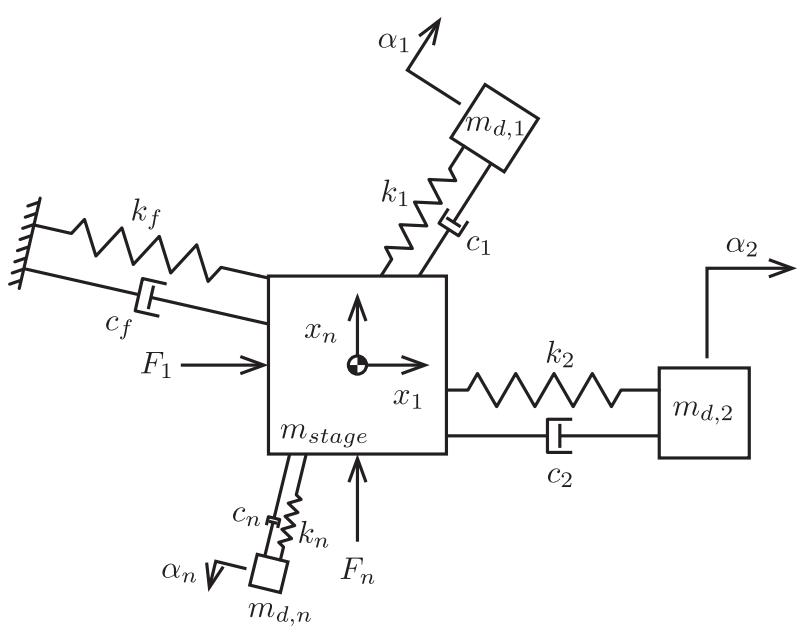

Fig. 5. Simple example of the modeling of a dynamic link disturbance with multiple mass-spring systems. Every disturbance $n$ is modeled by a mass $m_{n}$, stiffness $k_{n}$ and damping $c_{n}$. The stage mass $m_{\text {stage }}$ moving in directions $x_{n}$ is controlled by control forces $F_{n}$. The stage has rigid body stiffness and damping $k_{f}$ and $c_{f}$.

degrees of freedom $\alpha_{1}$ to $\alpha_{n}$, e.g. representing the free vibrating disturbance masses attached to the respective stage. The stage is connected to the fixed world by a certain stiffness $k_{f}$ and damping $c_{f}$.

The equations of motion of the system in Fig. 5 are

$\mathbf{M} \ddot{\mathbf{q}}+\mathbf{C} \dot{\mathbf{q}}+\mathbf{K q}=\mathbf{F}$,

with the vector of degrees of freedom $\mathbf{q}=[\mathbf{x} \boldsymbol{\alpha}]^{T}$, a mass matrix $\mathbf{M}$, the damping matrix $\mathbf{C}$, the stiffness matrix $\mathbf{K}$ and input forces $\mathbf{F}$. Eq. (1) is partitioned for the mechanical system of Fig. 5 as follows

$$
\left[\begin{array}{cc}
\mathbf{M}^{x x} & \mathbf{0} \\
\mathbf{0} & \mathbf{M}^{\alpha \alpha}
\end{array}\right]\left[\begin{array}{c}
\ddot{\mathbf{X}} \\
\ddot{\alpha}
\end{array}\right]+\left[\begin{array}{ll}
\mathbf{C}^{\alpha x} & \mathbf{C}^{\alpha \alpha} \\
\mathbf{C}^{\alpha x} & \mathbf{C}^{\alpha \alpha}
\end{array}\right]\left[\begin{array}{c}
\dot{\mathbf{X}} \\
\dot{\boldsymbol{\alpha}}
\end{array}\right]+\left[\begin{array}{ll}
\mathbf{K}^{x x} & \mathbf{K}^{\alpha \alpha} \\
\mathbf{K}^{\alpha x} & \mathbf{K}^{\alpha \alpha}
\end{array}\right]\left[\begin{array}{c}
\mathbf{X} \\
\boldsymbol{\alpha}
\end{array}\right]=\left[\begin{array}{c}
\mathbf{F}_{c} \\
\mathbf{0}
\end{array}\right],
$$

where $\mathbf{x}$ are the degrees of freedom of the controlled stage and $\boldsymbol{\alpha}$ the degrees of freedom of the disturbances. Furthermore, $\mathbf{M}^{x x}$ is the mass matrix of the directly controlled stage of the plant, $\mathbf{M}^{\alpha \alpha}$ the mass matrix of the disturbances acting on the controlled states. The coupling terms $\mathbf{M}^{\alpha x}$ and $\mathbf{M}^{\alpha \alpha}$ between the states $\mathbf{x}$ and $\alpha$ are considered to be zero. The matrices $\mathbf{C}^{x x}, \mathbf{C}^{x \alpha}, \mathbf{C}^{\alpha x}$ and $\mathbf{C}^{\alpha \alpha}$ make the damping matrix of the system. The stiffness matrices $\mathbf{K}^{\alpha x}, \mathbf{K}^{\alpha \alpha}, \mathbf{K}^{\alpha x}$ and $\mathbf{K}^{\alpha \alpha}$ make the stiffness matrix of the system. The control force input $\mathbf{F}_{c}$ only acts directly on the controlled degrees of freedom $\mathbf{x}$.

In Fig. 5, the total disturbance force $\mathbf{F}_{d}$ on the stage due to the modeled disturbances can be computed from the equations of motion (2)

$\mathbf{F}_{d}=\left[\begin{array}{ll}\mathbf{C}^{x x} & \mathbf{C}^{x \alpha}\end{array}\right]\left[\begin{array}{c}\dot{\mathbf{x}} \\ \dot{\boldsymbol{\alpha}}\end{array}\right]+\left[\begin{array}{ll}\mathbf{K}^{x x} & \mathbf{K}^{x \alpha}\end{array}\right]\left[\begin{array}{l}\mathbf{x} \\ \boldsymbol{\alpha}\end{array}\right]$.

Note that the computation for the disturbance force by Eq. (3) is only valid for systems, like in Fig. 5, where the coupling via the mass matrices $\mathbf{M}^{\alpha x}$ and $\mathbf{M}^{\alpha \alpha}$ is zero. In general this may not be the case and it should be verified if the simplification is allowed.

\section{Estimation of the disturbance force}

The (linearized) state space description of the system in Fig. 5 is

$\dot{\mathbf{x}}_{s s}=\mathbf{A}_{s s} \mathbf{x}_{s s}+\mathbf{B}_{s s} \mathbf{u}_{s s}+\mathbf{v}_{s s}$,

$\mathbf{y}=\mathbf{C}_{s S} \mathbf{x}_{s S}+\mathbf{w}_{s S}$,

where $\mathbf{A}_{s s}$ is the system matrix, $\mathbf{B}_{s s}$ is the input matrix, $\mathbf{C}_{s s}$ is the output matrix, $\mathbf{x}_{s s}$ the state vector and $\mathbf{u}_{s s}$ the input, or control, vector.
The vector $\mathbf{v}_{s s}$ is an unknown input noise (different from the disturbances already accounted for within the model) and $\mathbf{w}_{s s}$ the measurement noise.

The state space matrices $\mathbf{A}_{s s}$ and $\mathbf{B}_{s s}$ are defined using the equations of motion (1) and (2)

$$
\mathbf{A}_{s s}=\left[\begin{array}{cc}
\mathbf{0} & \mathbf{I} \\
-\mathbf{M}^{-1} \mathbf{K} & -\mathbf{M}^{-1} \mathbf{C}
\end{array}\right],
$$

and

$$
\mathbf{B}_{s s}=\left[\begin{array}{c}
\mathbf{0} \\
\mathbf{M}^{x x^{-1}} \\
\mathbf{0}
\end{array}\right]
$$

With the vector of degrees of freedom $[\mathbf{x} \boldsymbol{\alpha}]^{T}$ from the equations of motion (2), the state vector is defined as

$\mathbf{x}_{s S}=\left[\begin{array}{c}\mathbf{x} \\ \boldsymbol{\alpha} \\ \dot{\mathbf{x}} \\ \dot{\alpha}\end{array}\right]$.

The disturbance force $\mathbf{F}_{d}$ of Eq. (3) is expressed as

$\mathbf{F}_{d}=\mathbf{K}_{s s} \mathbf{x}_{s s}$

where $\mathbf{K}_{s s}$ is the matrix

$\left[\begin{array}{llll}\mathbf{K}^{x x} & \mathbf{K}^{x \alpha} & \mathbf{C}^{x x} & \mathbf{C}^{x \alpha}\end{array}\right]$.

In most systems not all states $\mathbf{x}_{s s}$ are directly measured. A practical method of estimating the states of a system is using an observer or estimator [9] given in Fig. 6. With the observer an estimation of $\tilde{\mathbf{x}}_{s s}$ can be made to acquire all states. An estimation of the disturbance force $\tilde{\mathbf{F}}_{d}$ is made with

$\tilde{\mathbf{F}}_{d}=\mathbf{K}_{s s} \tilde{\mathbf{x}}_{s S}$,

where $\mathbf{K}_{s s}$, as defined by (9), is used as the state feedback gain of the observer. Note that the assumption of $\mathbf{M}^{\alpha \alpha}$ and $\mathbf{M}^{\alpha \alpha}$ being zero allows the direct estimation of $\tilde{\mathbf{F}}_{d}$ by the observer.

With the estimation of the disturbance $\tilde{\mathbf{F}}_{d}$ the signal added to the control signal is

$\mathbf{u}_{d}=-\tilde{\mathbf{F}_{d}}$.

When the estimation and the modeling of $\mathbf{F}_{d}$ is accurate, the observer will exactly compensate the disturbance introduced by the dynamic links. Note that all stability requirements for observer apply. The method for designing the state feedback $\mathbf{K}_{s s}$ as outlined will not guarantee a stable feedback system, depending on the mechanics of the model. Therefore the eigenvalues of

$\mathbf{A}_{s s}-\mathbf{B}_{s s} \mathbf{K}_{s s}$

should be on the left half plane for stability.

\section{Disturbance attenuation as an internal model control problem}

When using the state feedback $\mathbf{K}_{s s}$ as defined in Eq. (9) with an actual plant, the following should be taken into account. The actual disturbance can differ from the modeled disturbance of Eq. (3). This could be due to modeling errors, position dependent and other non-linear dynamics not accounted for; such as coupling via the mass matrix. In order to take these into account in the analysis, they are included in the an unknown input noise $\mathbf{v}_{s s}$ and an unknown measurement noise $\mathbf{w}_{s s}$ as depicted in the structure of Fig. 6.

The observer structure of Fig. 6 can be analyzed as an internal model problem. This will show that the disturbance attenuation 


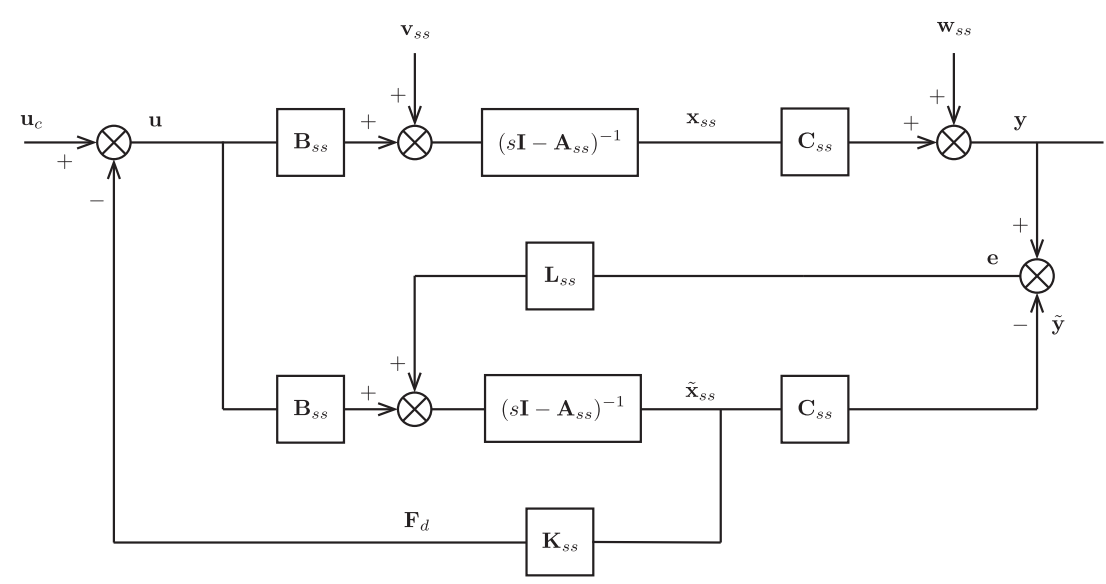

Fig. 6. Observer structure. Note that the encompassing control loop of Fig. 3 is left out. The observer has a gain $\mathbf{L}_{s s}$ to account for estimation errors due to input noise $\mathbf{v}_{s s}$ and output disturbances $\mathbf{w}_{s s}$. With the state feedback gain $\mathbf{K}_{s s}$ the control signal $\mathbf{u}_{d}$ is calculated.

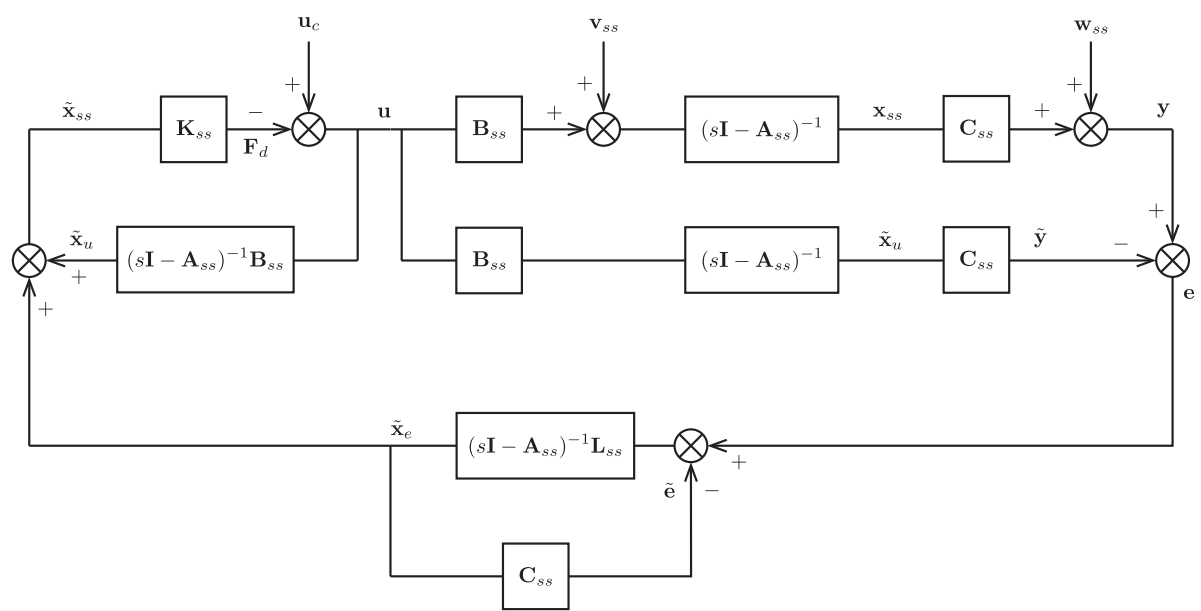

Fig. 7. IMC structure of the observer equivalent to Fig. 6.

problem with an state feedback design as outlined in the previous sections is equivalent to designing an observer with robustness for modeling errors.

The observer structure in Fig. 6 can be redrawn in the equivalent IMC structure as shown in Fig. 7 [8]. This structure separates the state estimation $\tilde{\mathbf{x}}_{s s}$ to

$\tilde{\mathbf{x}}_{s s}=\tilde{\mathbf{x}}_{u}+\tilde{\mathbf{x}}_{e}$

where $\tilde{\mathbf{x}}_{u}$ is the state estimation based on the control input to the observer

$\tilde{\mathbf{X}}_{u}=\left(s \mathbf{I}-\mathbf{A}_{s s}\right)^{-1} \mathbf{B}_{s s} \mathbf{u}$

and $\tilde{\mathbf{x}}_{e}$ is the estimated error of the state estimation $\tilde{\mathbf{x}}_{u}$

$\tilde{\mathbf{x}}_{e}=\left(\mathbf{I}+\left(s \mathbf{I}-\mathbf{A}_{s s}\right)^{-1} \mathbf{L}_{s s} \mathbf{C}_{s s}\right)^{-1} \times\left(s \mathbf{I}-\mathbf{A}_{s s}\right)^{-1} \mathbf{L}_{s s}$.

The separation between estimation based on the control input and estimation error can also be made for the disturbance force by substituting Eq. (13) into Eq. (13)

$\tilde{\mathbf{F}}_{d}=\mathbf{K}_{s s} \tilde{\mathbf{x}}_{u}+\mathbf{K}_{s S} \tilde{\mathbf{x}}_{e}$

where $\mathbf{K}_{s s} \tilde{\mathbf{x}}_{u}$ is the assumed disturbance force based on the equations of motion (2) and $\mathbf{K}_{s s} \tilde{\mathbf{x}}_{e}$ an estimation of the error between the actual and modeled disturbance force.
Defining a nominal plant $\mathbf{P}_{n}$ as consisting only the stage mass $m_{\text {stage }}$, without any disturbances, both modeled and unknown, the total input force $\mathbf{u}_{n}$ to that nominal plant can be defined as

$\mathbf{u}_{n}=\mathbf{u}+\mathbf{K}_{s s} \tilde{\mathbf{x}}_{u}+\boldsymbol{\delta}$,

being the complete control signal $\mathbf{u}$ itself, the force by the modeled disturbance $\mathbf{K}_{s \boldsymbol{S}} \tilde{\mathbf{x}}_{u}$ and an unknown disturbance force $\boldsymbol{\delta}$. Substituting Eq. (14) into (17)

$\mathbf{u}_{n}=\left[\mathbf{I}+\mathbf{K}_{s s}\left(\mathbf{s} \mathbf{I}-\mathbf{A}_{s s}\right)^{-1} \mathbf{B}_{s s}\right] \mathbf{u}+\boldsymbol{\delta}$,

enables to write the transfer of the actual plant in Fig. 7 as

$\mathbf{y}=\mathbf{P}_{n}\left\{\left[\mathbf{I}+\mathbf{K}_{s s}\left(\mathbf{s} \mathbf{I}-\mathbf{A}_{s s}\right)^{-1} \mathbf{B}_{s s}\right] \mathbf{u}+\boldsymbol{\delta}\right\}$,

and the transfer of the model as

$\mathbf{y}=\mathbf{P}_{n}\left[\mathbf{I}+\mathbf{K}_{s s}\left(s \mathbf{I}-\mathbf{A}_{s s}\right)^{-1} \mathbf{B}_{s s}\right] \mathbf{u}$.

In Fig. 7, the plant can be substituted by the structure in Eq. (19), and the model replaced with the structure in Eq. (20), creating Fig. 8. Note that the original noise input $\mathbf{v}_{s s}$ of the observer is now included with $\delta$.

This structure shows that the estimation of the disturbance forces $\mathbf{K}_{s s} \tilde{\mathbf{x}}_{u}$ will cancel out the known part of the disturbances acting on the nominal plant $\mathbf{P}_{n}$. From a feedback and feed forward controller point of view (Fig. 9) the observer adds resonances and 


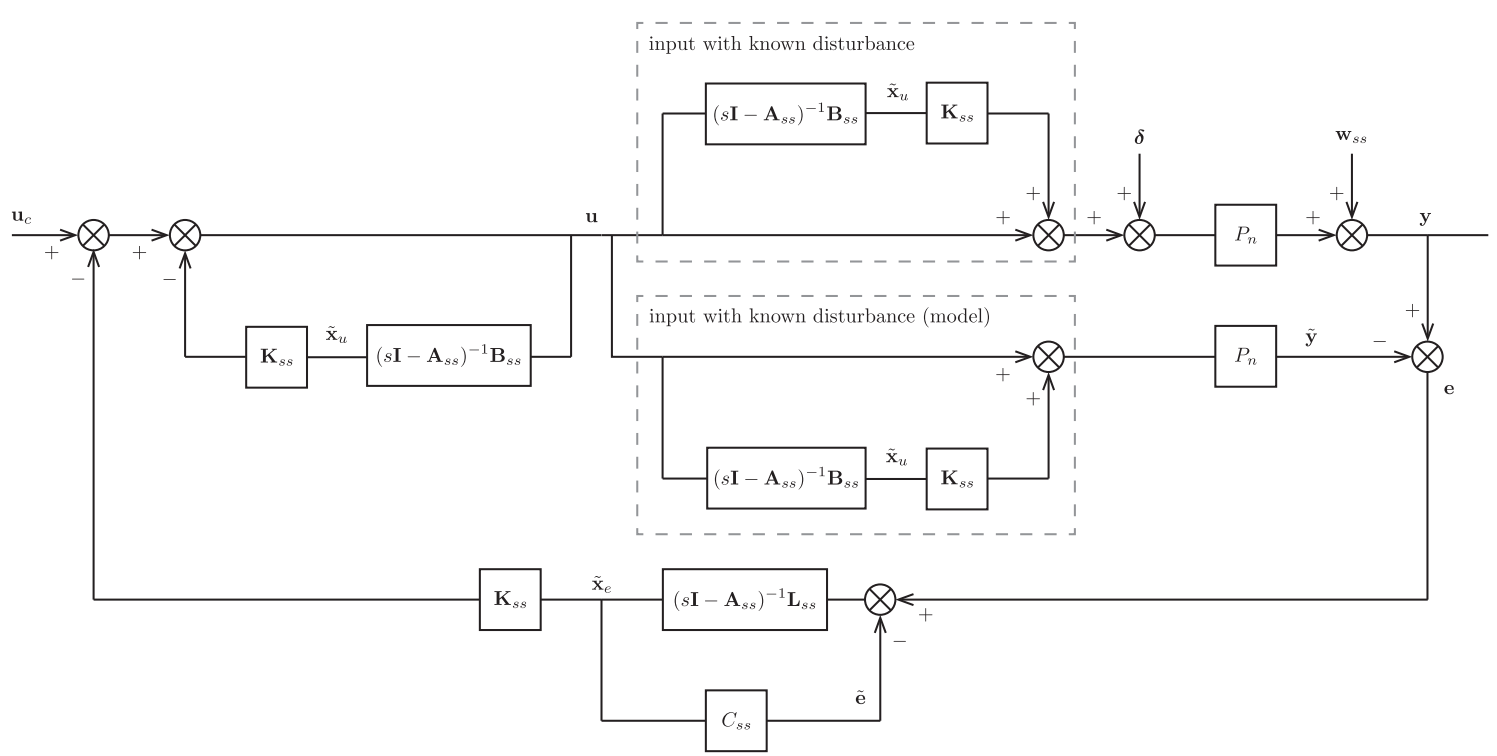

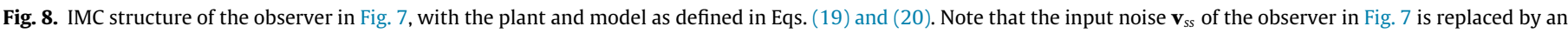
input noise $\delta$ to the nominal system $\mathbf{P}_{n}$.
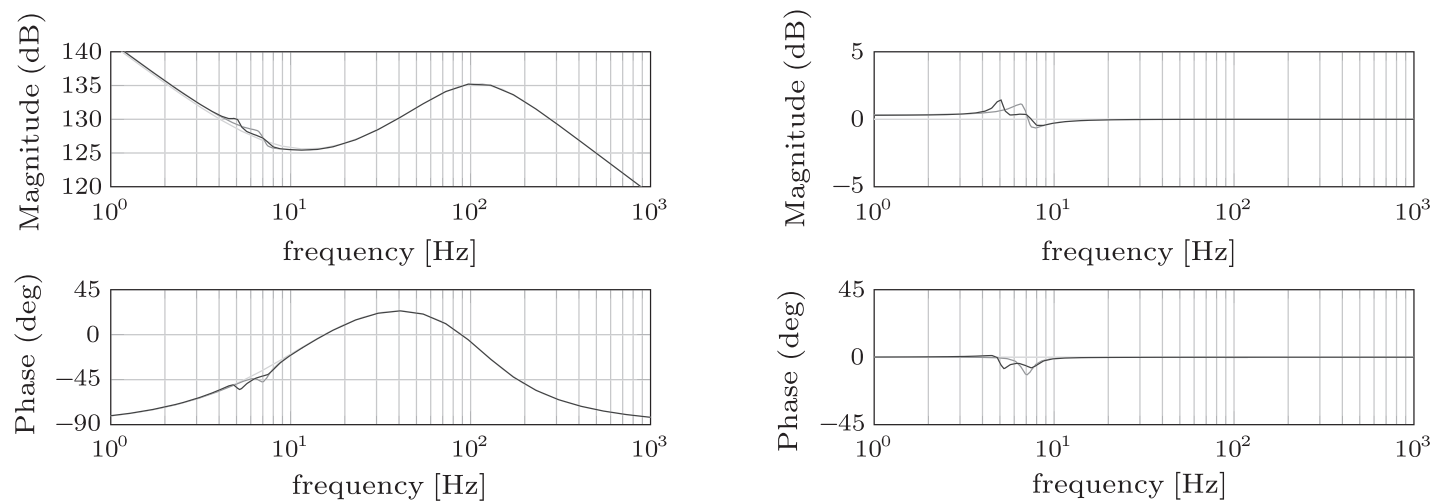

(a) Bode plot of the equivalent feedback controller.

(b) Bode plot of the equivalent feed forward controller.
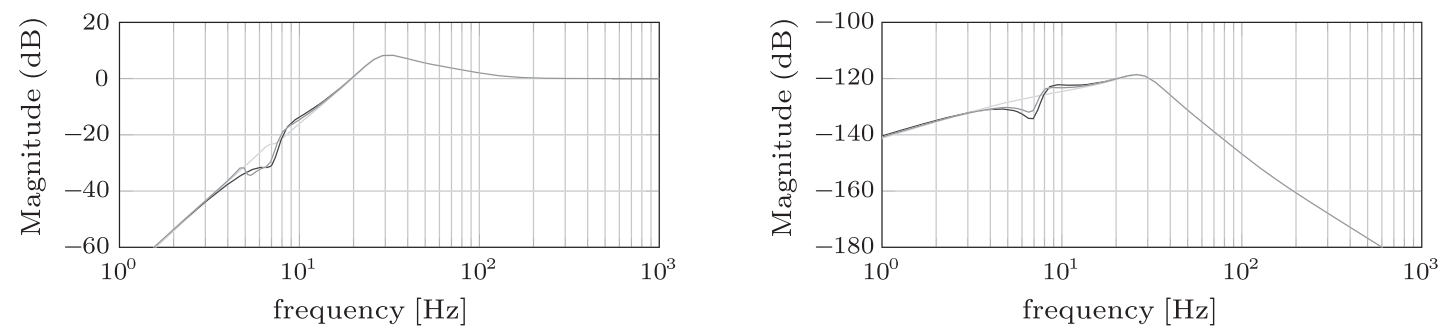

(c) Bode plot of the equivalent sensitivity.

(d) Bode plot of the equivalent process sensitivity.

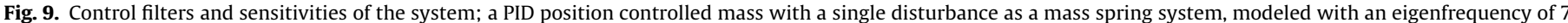

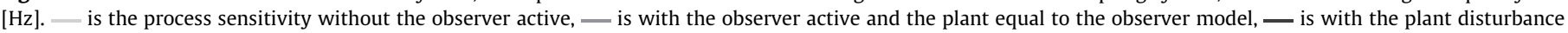

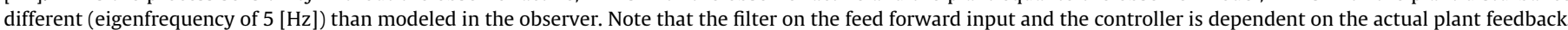

anti-resonances in both the feedback and feed forward control which match the anti-resonances and resonances of the modeled mass-spring disturbances. In the figures also the frequency responses of the Sensitivity and Process Sensitivity with a changed resonance $(5 \mathrm{~Hz}$ instead of $7 \mathrm{~Hz}$ ) are shown. Note that both the feedback and feed forward frequency responses have adapted to the change, which cannot be achieved with static feedback and feed forward loop shaping.

If the disturbance dynamics of the plant are fully known and described by equations of motion (2), the controller only needs to deal with the nominal plant $\mathbf{P}_{n}$. However, in practice modeling errors will exist, thus with the estimation of $\mathbf{K}_{s s} \tilde{\mathbf{x}}_{e}$ the model mismatch $\delta$ should be attenuated to achieve robustness.

Comparing the attenuation of the model mismatch $\delta$ in Fig. 8 to the attenuation of the full disturbance $\mathbf{d}$ in Fig. 4, shows the equivalence of the structure with the DEF. The filter $\mathbf{Q}$ and inverse model $\mathbf{P}_{n}^{-1}$ of the DEF is substituted by the feedback filter of the IMC structure, Eq. (15).

The purpose of the filter $\mathbf{Q}$ and inverse plant $\mathbf{P}_{n}^{-1}$ of the DEF in Fig. 4 and the feedback filter of Eq. (15) of the observer in Fig. 8 
is similar. Both should make an estimation $\tilde{\boldsymbol{\delta}}$ or $\tilde{\mathbf{d}}$ of the unknown input disturbance $\boldsymbol{\delta}$ or $\mathbf{d}$, respectively.

However, the model should match the plant as good as possible. Therefore the frequency content of $\delta$ originating from modeling errors is small compared to the frequency content of $\mathbf{d}$ if a DEF would be used for disturbance attenuation. The amount of attenuation of $\boldsymbol{\delta}$ can therefore be less than the amount of attenuation of $\mathbf{d}$ with a DEF. Compared to a DEF [4], this will limit sensitivity peaking by the observer as shown in Fig. 9(d) and (c). Therefore, disturbances can be attenuated in the frequency region near the sensitivity peak around the bandwidth of the control system.

\section{Achieving robustness for dynamic link modeling errors}

As the observer is designed to only attenuate specific modeled disturbances, the feedback filter of Eq. (15) should only try to estimate the disturbance force error in frequency ranges associated with the modeled disturbances.

If there is a disturbance force estimation error $\mathbf{K}_{s s} \tilde{\mathbf{x}}_{e}$, the assumption can be made that unknown extra, basically random, disturbance forces are acting on the plant that are not accounted for in the model. The effect of these forces on the estimation of the states $\mathbf{x}_{s s}$ of the observer is described by input noise vector $\mathbf{v}_{s s}$ in Fig. 6, and can be determined using the equations of motion

$$
\left[\begin{array}{cc}
\mathbf{M}^{x x} & \mathbf{0} \\
\mathbf{0} & \mathbf{M}^{\alpha \alpha}
\end{array}\right]\left[\begin{array}{c}
\ddot{\mathbf{x}} \\
\ddot{\boldsymbol{\alpha}}
\end{array}\right]+\left[\begin{array}{cc}
\mathbf{C}^{\alpha x} & \mathbf{C}^{\alpha \alpha} \\
\mathbf{C}^{\alpha x} & \mathbf{C}^{\alpha \alpha}
\end{array}\right]\left[\begin{array}{c}
\dot{\mathbf{x}} \\
\dot{\boldsymbol{\alpha}}
\end{array}\right]+\left[\begin{array}{cc}
\mathbf{K}^{x x} & \mathbf{K}^{\alpha \alpha} \\
\mathbf{K}^{\alpha x} & \mathbf{K}^{\alpha \alpha}
\end{array}\right]\left[\begin{array}{c}
\mathbf{x} \\
\boldsymbol{\alpha}
\end{array}\right]=\left[\begin{array}{c}
\mathbf{F}_{c} \\
\mathbf{0}
\end{array}\right]+\left[\mathbf{V}_{s s}\right] .
$$

Note that the forces $\mathbf{v}_{s s}$ have an initially unknown amplitude, however because this force should ideally be due to a model mismatch (e.g. a wrong stiffness $k_{n}$ or damping $c_{n}$ in the model of Fig. 5) an initial guess can be made.

With Eq. (21) and the state space matrices (5) and (6), the full observer model can be constructed:

$\dot{x}_{s s}=\left[\begin{array}{cc}\mathbf{0} & \mathbf{I} \\ -\mathbf{M}^{-1} \mathbf{K} & -\mathbf{M}^{-1} \mathbf{C}\end{array}\right] \mathbf{x}_{s s}+\left[\begin{array}{c}\mathbf{0} \\ \mathbf{M}^{x x^{-1}} \\ \mathbf{0}\end{array}\right] \mathbf{u}+\left[\begin{array}{c}\mathbf{0} \\ \mathbf{M}^{-1} \mathbf{v}_{s s}\end{array}\right]+\boldsymbol{w}_{s s}$,

where $\mathbf{w}_{s s}$ denotes the measurement noise vector.

With in optimization technique like Kalman filtering theory [9] the observer gain $\mathbf{L}_{s s}$ can then be determined using the solution of the Ricatti equation

$\dot{\mathbf{P}}=\mathbf{P A}+\mathbf{A P}+\mathbf{R}_{v}-\mathbf{P C}^{T} \mathbf{R}_{w}^{-1} \mathbf{C P}$

with the input noise covariance matrix

$\mathbf{R}_{v}=E\left(\mathbf{v}_{s s} \mathbf{v}_{s s}^{T}\right)$

and the measurement noise covariance matrix

$\mathbf{R}_{w}=E\left(\mathbf{w}_{s s} \mathbf{w}_{s s}^{T}\right)$.

The measurement noise covariance matrix can be determined by sensor noise measurements and is basically a given system property. The input noise covariance matrix $\mathbf{R}_{v}$ is assumed to be an function of the amplitude of random disturbance forces and can also be determined experimentally by varying the amplitude of applied input noise.

\section{Experimental setup}

To verify the method of attenuating dynamic link disturbances a test has been carried out on an actual stage of a lithography machine. The goal of the test was to see if the error introduced by the disturbance of the cable schlepp vibrations on the long

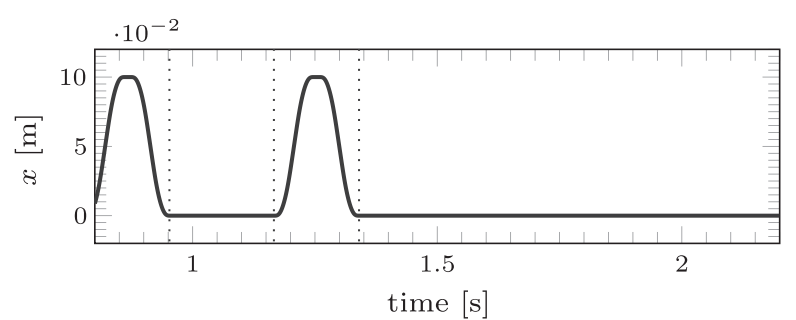

Fig. 10. Setpoint applied to the long stroke in $x$-direction. Two small moves are carried out after which the long stroke is held steady by the position controller.

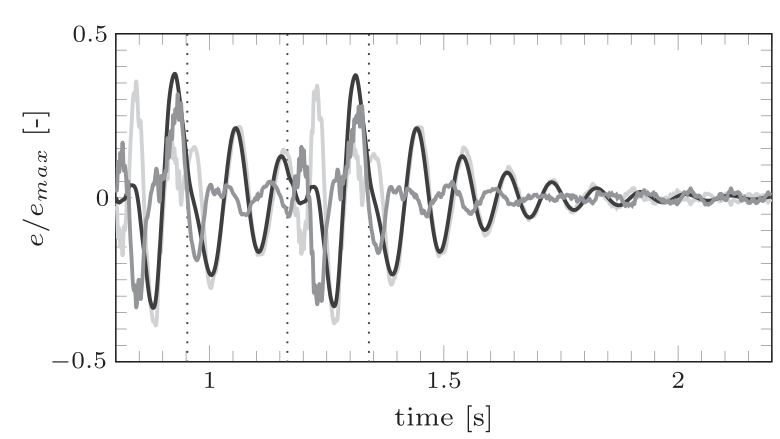

Fig. 11. Closed loop error — measured, — based on the model, — difference. The setpoint used is given in Fig. 10. Note that the errors are normalized.

stroke of the wafer stage could be reduced. The movement of the long stroke that has been carried out during the tests is according to a the set-point that results in a double back and forth movement. The position as a function of time is depicted in Fig. 10.

The model that is used for the observer is based on a the first cable schlepp vibration mode and is modeled as a mass-springdamper system acting on the long stroke, with one disturbance degree of freedom. Using measurement data of the lithography machine, the properties of the model were fitted to the dynamics of the actual stage and cable schlepp at the location $x=0$.

Fig. 11 shows the comparison of the closed loop position error from measurements to the closed loop position error as simulated with the model. When the reference point is zero, the stage is held steady by the controller and it appears that the error indeed resembles the response by a simple mass-spring-damper system. A reasonable fit between the error from simulations and measurements was achieved. During the acceleration phases however, the error is not predicted well by a simple mass-spring system, due to the nonlinear behavior of the cable schlepp.

The observer was tested using the same set-point profile. In order to test the robustness of the observer for position dependent and non-linear dynamics, the moves were carried out at three different locations $x$ of the long stroke as shown in Fig. 12. Fig. 13(a) shows the long stroke position error for these locations $x$ of the stage. The position error is dominated by the cable schlepp vibration. Comparing the steady state errors (from $t>1.35$ [s]) of the three positions shows that the cable schlepp does react similar fashion for each location, but with quite some variation in amplitude. However during acceleration phases the position error is very different in frequency and amplitude for each of the three locations.

\section{Experimental results}

In Fig. 13(c) the position error of the long stroke at $x=0[\mathrm{~mm}]$ is given. The differences in the error with the observer on and off are clearly visible. When the long stroke motion is finished, the 

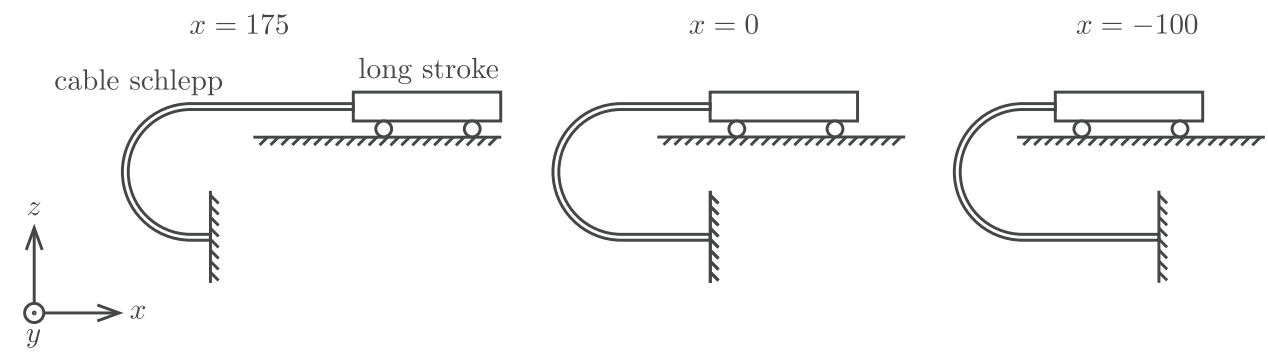

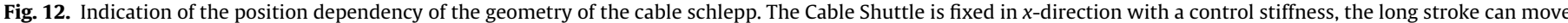
in the $x$-direction with a given position setpoint.

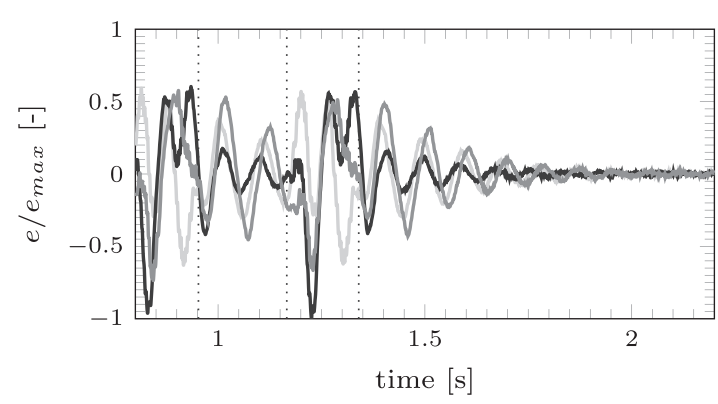

(a) Position errors of a long stroke without the disturbance observer active. The position of the long stroke is $-x=0[\mathrm{~mm}],-x=-100[\mathrm{~mm}]$ and $-x=175[\mathrm{~mm}]$.

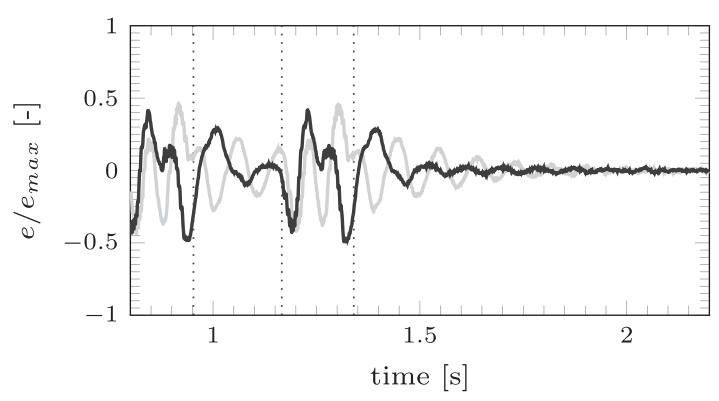

(c) Position error of a long stroke move at $x=0[\mathrm{~mm}] . \quad$ without observer, — with observer.

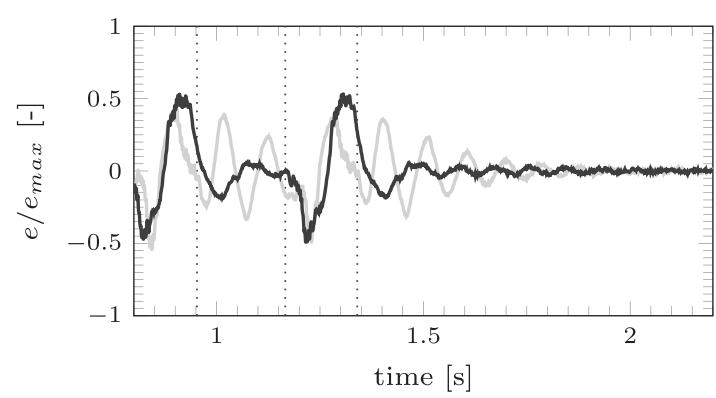

(b) Position error of a long stroke move at $x=175[\mathrm{~mm}]$. without observer, - with observer.

Fig. 13. Position error of the long stroke in $x$-direction at different $x$-locations. Note that the errors are normalized.

disturbance introduced by the cable schlepp is rapidly reduced. The double back and forth movement shows that the transient of the first move does not significantly affect the second move; the excitation of the second move is dominant over the transient behavior after the first move and the errors during the second move are almost identical to the errors during the first move.

The error in the experiment with the observer enabled reduces to near zero directly. This shows that observer seems to counter the disturbance forces based on state estimation of both the plant states $\mathbf{x}$ and disturbance states $\boldsymbol{\alpha}$. A similar result is obtained for the stage position at $x=175[\mathrm{~mm}]$ in Fig. 13(b). When the stage stands still, the error is rapidly reduced with the disturbance observer. This shows some robustness to different disturbance dynamics as shown in Fig. 13(a). However, the peak error during and just after acceleration is larger than without the disturbance observer, which indicates that the state estimation by the observer during stage moves is not sufficiently accurate. Also the dynamics at $x=0[\mathrm{~mm}]$ and $x=175[\mathrm{~mm}]$ are still quite comparable. This is not the case with the long stoke at $x=-100[\mathrm{~mm}]$, shown in Fig. 13(d). The cable schlepp reacts very different from what is expected based on the model, the disturbance observer does not give any meaningful attenuation of the disturbance, and the peak error during acceleration is twice as large.

The results indicate that the observer is not able to estimate the states of the disturbance during the acceleration and deceleration phases of the long stroke moves. A possible cause for the mismatch between the modeled and the actual dynamics is that the model and state feedback is based on the assumption that the disturbances are purely a result of dynamics based on a mass-springdamper structure like in Fig. 5 while the actual dynamic behavior of the cable schlepp is not. Another cause for the mismatch may be in the fact that there are no position, velocity and acceleration 
dependent dynamics taken into account in the linear observer model. Given the non-linear shape of the cable schlepp, it is to be expected that these dynamics may play a significant role especially at high accelerations and velocities.

\section{Conclusions}

The disturbance observer for dynamic links as introduced in this paper shows promising results for attenuation disturbances of a cable schlepp on a wafer stage.

The information gathered by modeling the mechanics of dynamic links can be used directly to construct a disturbance observer, which extends the UIDO with the information of the dynamic links such that the disturbance is not an independent augmented model, but integrated with the plant.

Rewriting the observer structure into an internal model control problem shows that including the modeled disturbance the disturbance observer problem is equivalent to designing an observer with robustness for modeling errors.

Robustness to modeling errors is given by the design of the observer feedback gain, which limits the peaking caused by the observer on the sensitivity of the error to the input disturbance. This enables the attenuation of disturbances closer to the bandwidth of the controlled system.

The advantage of the presented implementation of the disturbance observer over loop shaping and feed forward frequency input shaping is that only a single design effort for both feedback and feed forward control is needed.
An experiment on an actual lithography machine has shown that the disturbance observer as presented does attenuate the disturbances introduced by a cable schlepp. But in order to improve the performance, a better match between the actual plant and the model should be achieved, especially of the non-linear dynamics of the cable schlepp.

\section{References}

[1] Rankers AM. Machine dynamics in mechatronic systems: an engineering approach. PhD thesis, University of Twente; June 1997.

[2] de Roover D, Sperling FB, Bosgra OH. Point-to-point control of a MIMO servomechanism. In: Proceedings of the 1998 American control conference, vol. 5 ; 1998. p. $2648-51$

[3] Johnson CD. Accommodation of external disturbances in linear regulator and servomechanism problems. IEEE Trans Autom Control 1971;16(6):635-44.

[4] Schrijver Erwin, Dijk Johannes van. Disturbance observers for rigid mechanical systems: equivalence, stability, and design. J Dynam Syst Measure Control 2002;124(4):539.

[5] Ohnishi K, Shibata M, Murakami T. Motion control for advanced mechatronics. IEEE/ASME Trans Mechatron 1996;1(1):56-67.

[6] Liu Chia-Shang, Peng Huei. Inverse-dynamics based state and disturbance observers for linear time-invariant systems. J Dynam Syst Measure Control 2002;124(3):375.

[7] Hoogerkamp M, Waiboer RR, Aarts RGKM Modeling of flexible non-linear dynamic links in nano-positioning motion systems. In: Proceedings multibody dynamics, ECCOMAS 2013, Zagreb, Croatia, July 01-14; 2013.

[8] Garcia Carlos E, Morari Manfred. Internal model control 1: a unifying review and some new results. Ind Eng Chem Process Des Dev 1982;21(2):308-23.

[9] John O'Reilly T. Observers for linear systems, mathematics in science and engineering, vol. 17. Academic Press; 1983. 